\title{
Abstracts
}

Monatsschr Kinderheilkd 2014 • 162:467-478

DOI 10.1007/s00112-014-3121-x

(c) Springer-Verlag Berlin Heidelberg 2014

\section{Abstracts der 83. Wissenschaftlichen Halbjahrestagung der Gesellschaft für Pädiatrische Onkologie und Hämatologie e. V. (GPOH)}

\author{
23./24. Mai 2014, \\ Charité - Campus Virchow-Klinikum, Berlin
}

Wissenschaftliche Leitung: Prof. Dr. Angelika Eggert

\section{Grußwort}

Sehr geehrte Leserinnen und Leser,

es ist uns eine Freude, Ihnen auch in dieser Ausgabe der Monatsschrift Kinderheilkunde Einblicke in aktuelle Themen der Kinderonkologie zu geben, die im Rahmen unserer Halbjahrestagung am 24./25.5.2014 in Berlin präsentiert und diskutiert werden.

Bei dieser Frühjahrstagung werden wir den Teilnehmern im Fortbildungsteil zunächst einen komprimierten Überblick über verschiedene Aspekte des Berufsfelds „Kinderonkologie“ geben. Im wissenschaftlichen Teil gibt es erstmalig einen State-ofthe-Art-Vortrag, der sich vor allem an jüngere Kolleginnen und Kollegen in der kinderonkologischen Weiterbildung richtet und das Thema „Wilms-Tumor“ behandelt, bevor es in die vertiefte Diskussion neuer Studienaspekte zu dieser Erkrankung geht. Im weiteren Verlauf der Tagung erwarten wir außerdem interessante neue Daten und Konzepte aus den Gebieten der akuten lymphoblastischen Leukämie, der Hirntumoren, der Sichelzellerkrankung und der Palliativmedizin. Auszüge aus diesen Innovationen finden Sie in der Auswahl der Abstracts in dieser Ausgabe.

Ich wünsche Ihnen viel Spaß beim Lesen dieser spannenden Abstracts!
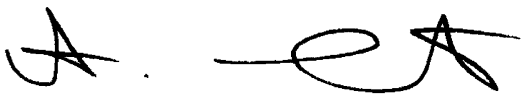

Prof. Dr. Angelika Eggert

Vorstandsvorsitzende GPOH 


\section{State of the Art: Wilms-Tumor}

\section{State of the Art: Wilms-Tumor}

\section{N. Graf}

Klinik für Pädiatrische Onkologie und Hämatologie, Universitätsklinikum des Saarlandes, Homburg/Saar

Nephroblastoma is the most common malignant renal tumour in childhood. Dramatic improvements in outcome have occurred over the last decades with survival rates over $90 \%$ in localized and $80 \%$ in metastatic tumours. Today treatments are based on multicentre trials and studies conducted primarily by the International Society of Paediatric Oncology (SIOP) Renal Tumour Study Group (RTSG) in Europe and the Children's Oncology Group [COG; formerly National Wilms Tumor Study Group (NWTSG)] in North America. The main objectives of these trials and studies are to treat patients according to well-defined risk groups in an attempt to achieve highest cure rates and lowest acute and late toxicities. The SIOP trials and studies largely focus on the issue of preoperative chemotherapy. More than 40 years after introducing preoperative chemotherapy for Wilms' tumour, the benefits of this approach are well known. The preoperative protocol results in easier operations with significantly fewer tumour ruptures during surgery and a favourable stage distribution. Acute toxicity and late effects are minimized without jeopardizing disease-free and overall survival. Clinical trials of today for Wilms' tumour seek additional risk factors to stratify and individualize treatment. These prognostic factors will improve the cure rates for highrisk patients by intensifying therapy and the quality of life for children with more favourable prognosis by lowering therapy to a minimum. In that respect diagnosis and treatment will be evaluated in all aspects including partial nephrectomy in unilateral disease in carefully selected patients according to clear and well-defined indications. Molecular genetic studies done in all patients will increase our understanding of Wilms' tumour, influencing treatment and outcome.

Acknowledgement: All nephroblastoma trials of GPOH are supported by: Deutsche Krebshilfe (Grant No.: 70-1899; 50-2709-Gr2).

\section{Nephroblastom}

\section{SIOP2001 Nephroblastoma Trial - update on results}

\section{R. Furtwängler', H. van Tinteren², N. Nourkami-Tutdibi', N. Graf' for the SIOP Renal Tumor Study Group}

'Pediatric Hematology and Oncology, Saarland University Hospital, Homburg/ Saar, ${ }^{2}$ Netherland's National Cancer Institute, Section of Biostatistics, Amsterdam, Netherlands

Since June 20014763 patients have been accrued to the trial, $26 \%$ coming from the GPOH. The randomization frequency of eligible intermediate risk nephroblastoma stage II and III was $67 \%$. Accrual was closed in December 2009 comprising 583 children. Both event-free (EFS) and overall survival (OS) for patients receiving AV were not inferior to AVD treated patients. Although a small excess of relapses was seen in the AV arm [Hazard ratio (HR) 1.57; 95\%CI 0.96-2.59) it was deemed acceptable since OS-curves were super imposable (HR 0.82; 95\%CI 0.35-1.92) and 5 -year-OS was $96 \%$.

2-year EFS and 5-year OS for the whole cohort are $95 \% / 97 \%$ for LR, $89 \% / 95 \%$ for IR and $73 \% / 75 \%$ for HR. EFS/OS for stage IV was $75 \% / 80 \%$. High Risk histology includes post-chemo blastema and diffuse anaplasia, suggesting an important role of blastema in the development of disease recurrence. We retrospectively analyzed the impact of absolute volume of blastema on relapse, yielding a positive correlation with risk of relapse: 2-year EFS and 6-year OS for patients having a volume of blastema of o $\mathrm{ml},<20 \mathrm{ml}, 20-100 \mathrm{ml}$ and $>100 \mathrm{ml}$ was $96 \% / 97 \%$, $92 \% / 96 \%, 78 \% / 91 \%$ and $68 \% / 76 \%$ respectively.

The vast majority of patients can now be treated without anthracyclines. They are restricted to high risk patients, thus minimizing the risk of cardiac sequelae in the majority of nephroblastoma patients. Further refining stratification using molecular markers will be the goal of the next nephroblastoma study. Stage IV local stage III and/or high risk histology stage III tumors are small groups that might still profit from increased treatment.

Acknowledgement: All nephroblastoma trials of GPOH are supported by: Deutsche Krebshilfe (Grant No.: 70-1899; 50-2709-Gr2).

\section{Update about classification of nephroblastoma}

\section{Leuschner}

Kiel Paediatric Tumor Registry, Dept. of Pediatric Pathology, University of Kiel

Nephroblastoma are classified according to the SIOP Renal Tumour Classification (2002) which is based on the classification of Beckwith and Palmer (1978). It was already realised in the SIOP9/GPOH study that the amount of blastema found after preoperative chemotherapy is an important risk factor for patients. Tumours containing more than two-third blastema in the viable part of the tumour in non-regressive nephroblastomas showed an association with a worse prognosis as compared to the tumours with less blastema. They are currently classified into the high risk group tumours.

A more detailed analysis of blastemal component revealed that the quantification of blastema after preoperative chemotherapy needs to be improved. Up to now the quantification is done semiquantitatively bases on one completely embedded slice of the tumor. To get a better quantification, a three-dimensional work-up is proposed. Still using one representative slice of the tumor to estimate the different components of the tumor, it is necessary to include the third dimension and the variability of tumor differentiation in the whole specimen to achieve the total volume of blastema. In addition markers for identification of blastema (e.g. SIX1, SIX2) need to be further analysed.

Most precise histopathological work-up of tumors will provide a better basis for understanding the prognostic value of blastemal differentiation in nephroblastoma.

\section{Molekularbiologie von Hochrisiko- Wilms-Tumoren des Blastemtyps}

\section{J. Wegert ${ }^{1}$, N. Ishaque 2 , R. Vardapour 1 , M. Kooß3, M. Gessler ${ }^{1}$}

'Theodor-Boveri-Institut/Biozentrum, Entwicklungsbiochemie, und Comprehensive Cancer Center Mainfranken, Julius-Maximilians-Universität, Würzburg, ${ }^{2}$ Heidelberg Center for Personalized Oncology, DKFZ-HIPO, Heidelberg, ${ }^{3}$ Pädiatrische Neuroonkologie, DKFZ, Heidelberg

Fragestellung. Wilms-Tumoren mit hohem Blastemanteil nach initialer Chemotherapie zählen zur Hochrisikogruppe mit ungünstiger Prognose, ebenso wie Tumoren mit diffuser Anaplasie. Während letztere oftmals Mutationen von TP53 aufweisen, sind die molekularen Charakteristika des Blastemtyps bislang unklar. Eine molekulare Definition dieser Hochrisikotumoren würde es erlauben, die Risikostratifizierung zu verbessern und solche Tumoren möglicherweise auch in Fällen ohne vorangegangene Chemotherapie zu erkennen. Zudem könnte die Identifizierung von sog. Driver-Mutationen und deregulierten Signalwegen unser Verständnis der Tumorentstehung verbessern und mithelfen, optimierte Therapiestrategien zu definieren.

Studiendesign. Wir haben 58 Wilms-Tumoren vom Blastemtyp identifiziert und durch umfassende Hochdurchsatzsequenzierung und Mikroarray-Analysen charakterisiert. Die Mehrzahl der Fälle stammt aus der GPOH/SIOP Wilms-Tumor-Studie, weitere von europäischen Partnern. Es wurden Exom-Sequenzierung, Genom-Sequenzierung, Tran- 
skriptomanalyse und miRNA-Analysen durchgeführt und die Daten mit bioinformatischen Methoden analysiert.

Ergebnisse. Die bislang in Wilms-Tumoren bekannten Mutationen in den Genen WT1, CTNNB1 und WTX wurden auch hier gefunden, allerdings nur in wenigen Fällen. Es ergaben sich jedoch klare Hinweise auf neuen Mutationen und Signalwege, die bevorzugt bei Tumoren mit hohem Blastemanteil zu finden sind. Diese Mutationen konnten in einem Screening von mehreren Hundert Tumoren aus der deutschen WilmsTumor-Studie validiert werden.

Schlussfolgerung. Die Hochdurchsatzsequenzierung und Transkriptomanalyse von Wilms-Tumoren ergaben neue Mechanismen der Tumorentstehung, die nun im Detail charakterisiert werden um den Pathomechanismus besser zu verstehen und mögliche diagnostische/ therapeutische Angriffspunkte zu identifizieren. Die Integration mit klinischen Daten wird zeigen, inwieweit solche Veränderungen bereits jetzt diagnostische Hilfestellungen bieten können.

\section{Nierenerhaltende und minimal-invasive Chirurgie beim Nephroblastom}

\section{D. von Schweinitz}

Kinderchirurgische Klinik der LMU, Dr. von Haunersches Kinderspital, München

Das nierenerhaltende Resezieren („nephron sparing surgery“, NSS) von Wilms-Tumoren (WT) ist für bilateralen Tumorbefall gut etabliert und sollte wenn immer möglich bei allen diesen Patienten durchgeführt werden. Bei unilateralen WT kommt die NSS nur in 10-15\% der Fälle in Betracht, wenn keine Einschränkung der Radikalität hingenommen werden soll. Sie kann bei diesen Patienten jedoch langfristig den Erhalt einer besseren Nierenfunktion ermöglichen. Insbesondere kommen hierfür WT in Betracht, die sich auf eine Hälfte einer Niere beschränken. Gerade bei Patienten mit Metastasen (Stadium IV), aber kleinem Primärtumor kann die NSS von besonderem Vorteil sein. Bei möglichem Lymphknotenbefall ist die Indikation zu NSS umstritten.

Die minimal-invasive Chirurgie (MIC) zur Resektion von WT erscheint attraktiv, weil sie eine schnellere Erholung der Patienten, eventuell weniger intestinale Verwachsungen und eine bessere Kosmetik erlaubt. Probleme hierbei sind jedoch die häufiger eingeschränkte Radikalität, die Schwierigkeit einer umfassenden Lymphknotendissektion vor allem hinter der Leber oder der Milz und dem Pankreasschwanz sowie zwischen V. cava und Aorta und der trotzdem immer noch notwendige offene Schnitt zur Bergung des Tumors. So kommt die MIC nur für kleine WT in Betracht, die zentral in einer Niere gelegen sind. Bei anderen lokal beschränkten WT sollte, wenn möglich, eher die (meist offene) NSS bevorzugt werden, weil die Patienten langfristig hiervon einen größeren Nutzen haben. Bei beiden Techniken muss immer die nötige Radikalität gewährleistet sein. Zur Beurteilung beider Techniken fehlen derzeit noch prospektive oder gar randomisierte Studien. Dieses Problem soll in der SIOP-RTSG Nachfolgestudie aufgegriffen werden.

\section{Concept of the upcoming Renal Tumour study and trial}

\section{N. Graf for the Renal Tumour Study Group}

Klinik für Pädiatrische Onkologie und Hämatologie, Universitätsklinikum des Saarlandes, Homburg/Saar

A new concept for renal tumours in childhood and adolescence is discussed within the SIOP Renal Tumour Study Group and based on results of former trials of SIOP and COG. The upcoming study and trial will recruit all patients with renal tumours in participating centres of SIOP-RTSG. An „umbrella“ protocol will cover all aspects of renal tumours, including logistics, legal and ethical aspects, data management, diagnostic procedures, pathology, general surgical, radiotherapeutic and chemotherapeutic aspects. Specific protocols will be provided for the treatment of patients with unilateral and localized nephroblastoma, metastasized nephroblastoma, bilateral diseases, non-Wilms tumors, relapsed tumors and nephroblastoma in adults. In patients with localized unilateral nephroblastoma the main objective is to collect biomaterial from every patient to define new biomarkers for a better stratification of treatment. Better methods for the calculation of the absolute volume of blastema will be developed and the retrospective results concerning the absolute blastemal volume will be validated without changing treatment compared to SIOP 2001. Patients with metastatic nephroblastoma will be randomized receiving different pre-operative chemotherapies to test if a carboplatin containing regimen results in higher response rates at the time of surgery and if anthracyclines can be further omitted in nephroblastoma. In bilateral diseases the collection of late effects is a main objective. Non-Wilms tumours (CCSK, RTK, RCC) will be treated within registries. Patients with relapses will be stratified according to their outcome into different treatment arms. A registry for adults with nephroblastoma will be set up. Nephron sparing surgery and minimally invasive surgery will be prospectively evaluated in all treatment groups. For each of the different treatment groups the concept will be presented in detail.

Acknowledgement: All nephroblastoma trials of GPOH are supported by: Deutsche Krebshilfe (Grant No.: 70-1899; 50-2709-Gr2).

\section{Akute lymphoblastische Leukämie}

\section{Concurrent deletions of IKZF1 and PAX $5, C_{1} \mathrm{CKN}_{2} \mathrm{~A}$, CDKN2B or CRLF2 (IKZF1plus) confer a very poor prognosis in pediatric acute lymphoblastic leukemia}

\section{E. Dagdan', M. Zaliova', M. Zimmermann', P. Dörge ${ }^{3}$, A. Möricke ${ }^{3}$, A. Teig-} ler-Schlegel ${ }^{4}$, R. Koehler ${ }^{5}$, C.R. Bartram ${ }^{5}$, J. Alten ${ }^{3}$, D. Schewe ${ }^{3}$, C. Kratz', R.S. Houlston ${ }^{6}$, M. Schrappe ${ }^{3}$, G. Cario ${ }^{3}$, M. Stanulla ${ }^{1}$

'Pediatric Hematology and Oncology, Hannover Medical School, ${ }^{2} \mathrm{CLIP}$ - Childhood Leukemia Investigation Prague, Department of Pediatric Hematology and Oncology, ${ }^{2}$ nd Faculty of Medicine, Charles University and University Hospital Motol, ${ }^{3}$ Department of Pediatrics, University Hospital Schleswig-Holstein, ${ }^{4}$ Oncogenetic laboratory, Pediatric Hematology and Oncology, University of Giessen, ${ }^{5}$ Department of Human Genetics, University of Heidelberg, ${ }^{6}$ Institute of Cancer Research Sutton, Division of Genetics and Epidemiology, Sutton

Study question. Recently, GATA3 was described as a new susceptibility locus for pediatric acute lymphoblastic leukemia (ALL, Blood 2013). The variant GATA3 allele ( $\mathrm{rs} 3824662)$ was found to be specifically associated with B-other ALL (B-lineage ALL lacking recurrent chromosomal translocations and hyperdiploidy) and to confer a dismal outcome. Study aim was the analysis of somatic genetic aberrations describing B-other ALLs associated with $\mathrm{rs} 3824662$ to characterize the leukemia genetics underlying the prognostic germline GATA3 ALL risk-allele.

Study design. Clinical epidemiological analysis of 500 patients treated on trial AIEOP-BFM 2000 having information on rs3824662 genotype and leukemia genetic characteristics. Validation of results in an independent cohort.

Results. GATA3 rs3824662 genotype was negatively associated with hyperdiploidy and positively associated with prednisone poor-response, higher loads of minimal residual disease (MRD), and deletions of $I K Z F_{1}, P A X_{5}, C D K N_{2} A$ and $C D N_{2} B$. In single marker analyses, $I K Z F_{1}$ deletion was the strongest determinator of outcome. In $C D N_{2} A-$ and $\mathrm{CDKN}_{2} B$-deleted patients, the prognostic impact was restricted to those with homozygous deletions. When $I K Z F_{1}$ deletions were analyzed in the discovery and validation cohorts in combination with $P A X_{5}, C D K N_{2} A$ and $C D K_{2} B$ deletions, patients displaying an additional deletion to that of $I K Z F_{1}$ had the worst event-free survival (EFS) and highest cumulative incidence of relapse (CIR). We also analyzed $I K Z F_{1}$ deletion in association with $P A R 1$ deletions leading to $P_{2} R Y 8-C R L F 2$ fusion, 
which we previously described as prognostically relevant in our patient population (Blood 2010) and detected similar combinatorial effects as described above. Consequently, we defined a group by presence of $I K Z F_{1}$ deletion and at least one additional deletion in $P A X_{5}, C D K_{2} A$, $C D K N_{2} B$ or $P A R 1$. This group comprising $6 \%$ of B-lineage ALL patients was termed IKZFiplus and had a very poor clinical outcome: $5 \mathrm{y}$-EFS $55 \% \pm 0.07$ compared to $85 \% \pm 0.01$ in IKZFiplus-negatives $(\mathrm{p}<0.0001$ ); $5 \mathrm{y}-\mathrm{CIR} 42 \% \pm 0.07$ compared to $11 \% \pm 0.01$ ( $\mathrm{p}<0.0001)$. In multivariate analyses including MRD, slow early response, prednisone response, ETV6/RUNX1 status, and WBC ( $\geq 100,000 / \mu \mathrm{l}), I K Z F 1 p l u s$ displayed the highest hazard ratio for relapse (3.29; 95\% CI 2.01-5.38; $\mathrm{p}<0.001)$.

Conclusions. The previously described effect on clinical outcome of GATA $_{3}$ SNP rs3824662 is due to its association with distinct prognostic leukemic lesions. Combinatorial analysis of deletions in IKZF1, PAX5, $\mathrm{CDKN}_{2} \mathrm{~A}, \mathrm{CDKN}_{2} \mathrm{~B}$ and $\mathrm{PAR}_{1}$ allowed description of a very poor prognostic subgroup of ALL - termed IKZFiplus - with significantly worse outcome compared to the use of IKZF1 deletion or others as a sole marker. The definition of IKZFiplus is likely to aid in the practical implementation of newly detected markers for risk stratification in childhood ALL.

\section{Darstellung klonaler Evolution bei rezidivierter T-ALL mittels "whole exome sequencing" (WES) und zielgerichtetem "deep sequencing"}

\section{J.B. Kunz', T. Rausch'2, J.O. Korbel', A.E. Kulozik'}

${ }^{1}$ Zentrum für Kinder- und Jugendmedizin, INF ${ }^{430}$, Heidelberg, ${ }^{2}$ EMBL Heidelberg, Heidelberg

Hintergrund. Rezidive einer T-lymphoblastischen Leukämie (T-ALL) zeichnen sich oft durch eine ausgeprägte Therapieresistenz aus. Wir haben Proben vom Zeitpunkt der Ersterkrankung, der Remission und des ersten Rezidivs von 13 Patienten mit rezidivierter T-ALL mittels „whole exome sequencing" untersucht.

Methoden. Im Durchschnitt fanden sich 9,4 Punktmutationen („single nucleotide variants", SNV) und 2,3 kleine Insertionen oder Deletionen (Indels) in Proben von der Ersterkrankung und 19,6 SNVs und 4,2 Indels in der zugehörigen Rezidivprobe. Während nur etwa $30 \%$ der im Rezidiv nachweisbaren Mutationen sich schon in der Ersterkrankung fanden, blieben Amplifikationen und Deletionen, die wir mittels MLPA und auch WES nachwiesen, zwischen beiden Erkrankungszeitpunkten weitgehend konserviert. Bekannte und auch neu beschriebene leukämogene Mutationen fanden sich in mehr als einem Patienten, darunter Notch1, FBXW7, PHF6, WT1, PTEN, NRAS und STAT5B.

Ergebnisse. Die im WES identifizierten Mutationen haben wir in Proben von Ersterkrankung und Rezidiv mit hoher Sensitivität (10-3-10-2) mittels zielgerichtetem "deep sequencing“ ("coverage“ $>1000$ in $>90 \%$, median 6233) nach Anreicherung mittels HaloPlex (Agilent) untersucht. Damit können wir zwischen Mutationen, die schon bei Ersterkrankung nachweisbar waren und durch klonale Selektion angereichert wurden, und De-novo-Mutationen unterscheiden. Bei allen Patienten fand sich zumindest eine genetische Veränderung sowohl in der Ersterkrankung als auch im Rezidiv, alle Rezidive leiteten sich also von der ursprünglichen Leukämie ab. Die Anzahl der Mutationen, die ausgehend von einem kleinen Subklon in der Ersterkrankung im Hauptklon des Rezidivs nachweisbar waren, lag zwischen o und 4 pro Patient. Gleichzeitig fanden sich pro Patient zum Zeitpunkt des Rezidivs 1 bis 83 neu aufgetretene Mutationen. Unter den neu aufgetretenen Mutationen fanden sich auch aktivierende Mutationen in $\mathrm{NT}_{5} \mathrm{C}_{2}$, dem Gen für eine Nukleotidase, die am Abbau von Nukleosidanaloga beteiligt ist.

Schlussfolgerung. Diese Analysen zeigen, dass die rezidivierte T-ALL im Kindesalter in vielen Fällen durch die Akkumulation neuer Mutationen charakterisiert ist und dass es dadurch zu definierbaren, therapeutisch relevanten Resistenzmechanismen kommen kann.

\section{Minimal residual disease-based treatment is adequate for relapse-prone childhood acute lymphoblastic leukemia with an intrachromosomal amplification of chromosome 21: the experience of the ALL-BFM 2000 trial}

A. Attarbaschi', R. Panzer-Grümayer'², G. Mann', A. Möricke³, M. König², A. Mecklenbräuker ${ }^{2}$, A. Teigler-Schlegel', J. Bradtke5, J. Harbott', G. Göhring ${ }^{6}$, M. Stanulla ${ }^{7}$, M. Schrappe ${ }^{3}$, M. Zimmermann ${ }^{7}$, O.A. Haas ${ }^{1,2}$ on behalf of the Austrian and German ALL-BFM (Berlin-Frankfurt-Münster) Study Group

'Department of Pediatric Hematology and Oncology, St. Anna Children's Hospital, Department of Pediatrics and Adolescent Medicine, Medical University of Vienna, Vienna, Austria, ${ }^{2}$ Children's Cancer Research Institute (CCRI), Vienna, Austria, ${ }^{3}$ Department of Pediatric Hematology and Oncology, Children's University Hospital, University Hospital Schleswig-Holstein, Campus Kiel, Germany, ${ }^{4}$ Department of Pediatric Hematology and Oncology, Oncogenetic Laboratory, Justus-Liebig-University, Giessen, Germany, Institute of Pathology, JustusLiebig-University, Giessen, Germany, ${ }^{6}$ Institute of Cell and Molecular Pathology, Medical School of Hannover, Germany, ${ }^{7}$ Department of Pediatric Hematology and Oncology, Medical School of Hannover, Hannover, Germany

Background. Recently, the UK CCLG and COG reported that an intrachromosomal amplification of chromosome 21 (iAMP21) in acute lymphoblastic leukemia (ALL) loses its adverse prognostic impact with intensified therapy.

Methods. We evaluated the prognosis of iAMP21 among patients from the ALL-BFM (Berlin-Frankfurt-Münster) 2000 trial with 46 of 2637 (2\%) patients iAMP21+. Eight-year EFS (64 $\pm 8 \%$ vs. $81 \pm 1 \%$, p=0.0026) and cumulative incidence of relapse (CIR, $29 \pm 8 \%$ vs. $14 \pm 1 \%, \mathrm{p}=0.008)$ of the iAMP21 cases were significantly worse compared with non-iAMP 21 patients.

Results. Within the MRD low-risk group, iAMP21 cases $(n=14)$ had an inferior 8-year EFS ( $76 \pm 12 \%$ vs. $92 \pm 1 \%, \mathrm{p}=0.0081$ ), but no increased CIR ( $10 \pm 10 \%$ vs. $6 \pm 1 \%, \mathrm{p}=0.624)$. Within the MRD intermediate-risk group, iAMP21 cases $(n=27)$ had a worse 8 -year EFS $(56 \pm 11 \%$ vs. $78 \pm 2 \%$, $\mathrm{p}=0.0077)$ and CIR $(44 \pm 11 \%$ vs. $20 \pm 2 \%, \mathrm{p}=0.003)$ with $6 / 10$ relapses occurring after two years.

Conclusion. Conclusively, we believe that there is no necessity for enrolling all iAMP21 patients into the high-risk arm of ongoing ALL-BFM trials because MRD low-risk patients have a moderate relapse risk under current therapy. Whether the increased relapse risk in MRD intermediate-risk patients can be avoided by late treatment intensification remains to be answered by the AIEOP-BFM ALL 2009 trial using protracted pegylated L-asparaginase during delayed intensification and early maintenance.

\section{Asparagin-Depletion im Liquor unter pegylierter E.-coli-Asparaginase im Rahmen der AIEOP- BFM ALL 2009 Studie: Zwischenauswertung und praktische Konsequenz}

C. Lanvers-Kaminsky', S. Borghorst', G. Würthwein'², J. Gerss³, A. Möricke4, A. Ballerini ${ }^{5}$, C. Rizzari ${ }^{5}$, M. Schrappe ${ }^{4}$, J. Boos ${ }^{1}$

'Klinik für Kinder und Jugendmedizin, Pädiatrische Hämatologie und Onkologie, Universitätsklinikum Münster, Münster, ${ }^{2}$ Zentrum für Klinische Studien, Münster, ${ }^{3}$ Institut für Biometrie und Klinische Forschung, Münster, ${ }^{4} \mathrm{Klinik}$ für Allgemeine Pädiatrie - Hämatologie und Onkologie - Universitätsklinikum Schleswig Holstein, Kiel, ${ }^{5}$ University of Milano-Bicocca Department of Pediatric Hematology-Oncology, Monza, Italy

Ziel. In der AIEOP-BFM ALL 2009 Studie wird auf die Gabe nativer E.coli-ASE von vornherein zugunsten von pegylierte E.-coli-Asparaginase 
(PEG-ASE) verzichtet. Um die Auswirkungen der Umstellung auf die Intensität der ASE-Therapie zu erfassen, wurde die Umstellung durch das Monitoring von ASE-Aktivitäten im Serum und Asparagin(ASN)Konzentrationen im Liquor begleitet.

Methode. Zwischen Februar 2011 und der geplanten Zwischenauswertung im Oktober 2012 wurden insgesamt 1135 Liquorproben von $523 \mathrm{~Pa}$ tienten auf ihren ASN-Gehalt untersucht. Die Nachweisgrenze betrug o,2 $\mu \mathrm{mol} / \mathrm{l}$ und lag damit deutlich unter der in der klinischen Routine üblicherweise angewandten Aminosäurenanalyzer. Insgesamt wurden 625 Liquorproben von 393 Patienten nach PEG-ASE-Gabe in Protokoll I und 98 Liquorproben von 97 Patienten nach PEG-ASE-Gabe in Protokoll II untersucht. Aus den Therapieabschnitten mit intensivierter ASETherapie wurden 41 Proben von 24 Patienten im Protokoll IB-ASP+ und 37 Proben von 22 Patienten im Protokoll II-ASP+ analysiert.

Ergebnisse. Die Gabe von 250o U/m2 PEG-ASE führte zu einer erheblichen Reduktion der ASN-Konzentrationen im Liquor, die im Protokoll I bis zu 3 Wochen nach der Gabe von 2500 U/m2 PEG-ASE am Tag 26 andauerte. Allerdings blieben bei ca. 50\% der nach PEG-ASE Gabe untersuchten Liquorproben relevante ASN-Konzentrationen von $\geq 2 \mu \mathrm{mol} / \mathrm{L}$ nachweisbar. Unterhalb einer Serumaktivität von $\leq 100$ U/L PEG-ASE war nur in 10\% der Liquorproben kein ASN mehr nachweisbar. Bei Serumaktivitäten zwischen 100-1000 U/L lag der Anteil der vollständig depletierten Liquorproben zwischen 40 und 50\%. Lag die Serumaktivität der PEG-ASE über $1000 \mathrm{U} / \mathrm{L}$ war in mehr als $60 \%$ der Proben keine Asparaginase-Aktivität mehr nachweisbar. Nach der Gabe von 2500 U/ m2 PEG-ASE war in allen Therapieabschitten (PI, PII, PII-ASP+, PIB$\mathrm{ASP}+$ ) eine signifikante Reduktion der Asparagin-Konzentrationen im Liquor zu beobachten. Im Gegensatz zur Behandlung mit nativer ASE war trotz beachtlicher ASE-Aktivitäten im Serum bei einem Großteil der Patienten keine vollständige ASN-Depletion im Liquor erreichbar. Schlussfolgerung. Auf dieser Grundlage hat die Studienkommission im Oktober 2012 beschlossen, das Monitoring von Asparagin im Liquor zu beenden. Das Monitoring der ASE-Aktivitäten und der AsparaginaseAntikörper wird fortgeführt.

\section{MRD-based treatment intensification before HSCT in childhood relapsed ALL: Is MRD reduced and the survival finally improved?}

\section{Eckert', C. Chen-Santel', G. Henze', A. von Stackelberg}

'Charité - Universitätsmedizin Berlin, Berlin

In childhood relapsed acute lymphoblastic leukemia (ALL), several studies demonstrated the prognostic value of minimal residual disease (MRD) prior hematopoetic stem cell transplantation (HSCT). In the trial ALL-REZ BFM 2002, online MRD-monitoring has been performed in all high and in some intermediate risk relapses since 2010. MRD results during consolidation phase and before HSCT have been directly communicated to the treating centres and intensification chemotherapy courses were offered with the aim to reduce persisting MRD and improve survival. As treatment intensification different courses/drugs were recommended as Clofarabine/Cyclophosphamide/Etoposide, Daunoxome/ or Idarubicine/-Fudarabine/Cytarabine, Nelarabine alone or with Cyclophosphamide/Etoposide. The intervention group consists of 1st ALL relapses with persisting MRD at a level of $10-3$ at the end of consolidation and therefore received an intensification block before $\operatorname{HSCT}(n=30)$. The non-intervention group includes 1st relapses having persisting MRD at a level of $10-3$ at the end of consolidation and received a HSCT $(\mathrm{n}=52)$.

MRD reduction of at least one log step was achieved in $82 \%$ and two log steps in $63 \%$ of patients after intervention. Comparing clinical characteristics between the intervention and non-intervention group, there were no differences except of MRD before HSCT. There were no differences in the probability of disease-free survival $(51 \% \pm 14 \%$ vs $48 \% \pm 7 \%)$, the cumulative incidence of subsequent relapse ( $31 \pm 9 \%$ vs $42 \pm 7 \%)$ and of therapy related death $(4.6 \pm 4.6 \%$ vs $9.6 \pm 4.1 \%)$ between the intervention and non-intervention group. Considering only patients with an at least two $\log$ reduction of MRD after intervention $(n=17)$, the proportion of patients in continuous complete remission (59\%) was not higher than in the non-intervention group ( $48 \%, \mathrm{p}=0.44)$. In summary, persisting MRD can be efficiently reduced by intensified chemotherapy. However, this does not translate into improvement of outcome. MRD persistence after intensive conventional chemotherapy seems to identify particularly aggressive leukemias which are not successfully treated by further reduction of the tumour load. Therefore, targeted therapies need to be investigated and prospective controlled studies should be preferred to individualized interventions.

\section{HIT-MED}

\section{Medulloblastom mit niedrigem Risiko oder Standardrisiko: die Studien PNET4 und PNET5}

\section{Mynarek', K. von Hoff', R.D. Kortmann², S. Rutkowski' et al.}

'Universitätsklinikum Hamburg-Eppendorf, Klinik für Pädiatrische Hämatologie und Onkologie, Hamburg ${ }^{2}$ Universität Leipzig, Klinik für Radioonkologie, Leipzig

Hintergrund. Das Medulloblastom ist der häufigste hochmaligne Hirntumor bei Kindern und Jugendlichen und geht mit einer hohen krankheits- und therapiebedingten Morbidität einher. Durch eine Verringerung der Therapieintensität bei Patienten mit geringem Risiko für Rezidiv und/oder Tod anhand bei Diagnose zu bestimmender Risikofaktoren soll therapieassoziierte Toxizität vermindert werden.

Ergebnisse aus PNET4. In HIT2Ooo waren Alter und Metastasierung der einzigen zur Stratifizierung genutzten prognostischen Faktoren. Im Studienarm für nichtmetastasierte Medulloblastomen bei Patienten $>4$ Jahren und seiner internationalen Erweiterung (PNET4), konnte in der postoperativen Therapie kein Unterschied zwischen einer konventionell fraktionierten und einer hyperfraktionierten Radiotherapie gezeigt werden. Das Vorhandensein eines großen postoperativen Resttumors und eine Aktivierung des WNT-Pathways (WNT-Subtyp) wurden als unabhängige prognostische Faktoren für das Überleben nach Diagnose identifiziert.

Therapiekonzept PNET5. Basierend auf den neuen Risikofaktoren wird in der Studie PNET5 eine erweiterte Therapiestratifizierung genutzt. Als Medulloblastom mit niedrigem Risiko (LR) gilt jeder Patient ohne Metastasen (Mo), ohne postoperativen Resttumor (Ro) und mit WNTPathway-Aktivierung (WNT-pos) ohne Nachweis einer myc-Amplifikation (myc neg). Bei diesen Patienten wird ein EFS von ca. 90\% erwartet. WNT neg, myc neg Medulloblastome MoRo werden definiert als Standartrisiko (SR) mit einem erwarteten EFS von ca. 80\%. Die Initiierung von PNET5 ist für April 2014 geplant. Ziel der Studie ist zu zeigen, dass eine Therapiereduktion bei Patienten mit LR-Medulloblastom sicher ist. Hierfür wird in einem einarmigen Phase-II-Konzept die auf die Radiotherapie folgende Chemotherapie verkürzt und voraussichtlich die Dosis der kraniospinalen Bestrahlung auf 18 Gy reduziert. Bei Patienten mit SR-Medulloblastomen wird in einem konfirmatorischen Phase-III-Design randomisiert Carboplatin zusätzlich zur Radiotherapie geprüft.

Untersuchung der Spätfolgen. Neuropsychologische Untersuchungen an $137 \mathrm{PNET}_{4}$-Patienten aus vier europäischen Teilnehmerländern zeigen insgesamt eine verminderte geistige Leistungsfähigkeit im Vergleich zur Normalbevölkerung. Da diese Ergebnisse von großer Bedeutung sowohl für die Beurteilung einzelner Therapieelemente als auch für die Planung der individuellen Förderung der Patienten nach Therapieabschluss sind, ist eine systematische Testung von Hirntumorpatienten nach Therapieabschluss dringend notwendig.

Gefördert durch die Deutsche Kinderkrebsstiftung. 


\section{Update Biologie: Medulloblastom, ZNS-PNET, Ependymom}

\section{T. Pietsch}

Universitätsklinikum Bonn, Institut für Neuropathologie, Hirntumorreferenzzentrum der Deutschen Gesellschaft für Neuropathologie und Neuroanatomie

In den letzten Jahren konnten insbesondere durch Identifikation rekurrierender genomischer Veränderungen und durch Expressionsstudien wesentliche biologische Signalwege und prognostische Marker identifiziert werden, die eine präzise Stratifizierung pädiatrischer neuroonkologischer Patienten für eine risikoadaptierte Therapie erlaubt. Zurzeit werden verschiedene Methodiken hinsichtlich ihrer Robustheit, Spezifität und Sensitivität in der Bestimmung dieser Marker in der neuropathologischen Routinediagnostik an Studienkollektiven geprüft.

Klinische, histologische, genetische und biologische Studien haben gezeigt, dass das Medulloblastom aus Varianten besteht, welche sich hinsichtlich ihrer Ursprungszelle, Pathogenese und klinisch-pathologischen Eigenschaften unterscheiden. Die revidierte WHO-Klassifikation 2007 unterscheidet 5 Varianten, die aktuelle biologische KonsensusKlassifikation mindestens 4 Varianten, die sich in unterschiedlichen Expressions- und Methylierungsprofilen widerspiegeln. Die Histologie stellt einen unabhängigen prognostischen Parameter bei Kleinkindern mit Medulloblastom dar; desmoplastische oder extrem noduläre Histologien korrelieren mit günstigem klinischem Verlauf und zeigen eine pathologische Aktivierung des Hedgehog-Entwicklungssignalweges an, zumeist durch Mutationen der PTCH1-, SMOH- oder SUFUHGene. Wnt-Signalweg-Aktivierungen werden zumeist in ca. $15 \%$ der Medulloblastome bei älteren Kindern durch aktivierende Mutationen des CTNNB1-Gens verursacht, und sind in Standardrisiko Medulloblastompatienten mit günstigem Outcome assoziiert, sodass in der aktuellen SIOP-PNET-5 Medulloblastomstudie geprüft werden soll, ob bei diesen Patienten die Therapie ähnlich wie die Kleinkindern mit desmoplastisch/nodulären Histologien reduziert werden kann. Die häufigsten chromosomalen Veränderungen bei non-Hedgehog, non-WNTMedulloblastomen betreffen Zugewinne des Chromosomenarms 17q, in einem Teil der Fälle als Isochromosom 17q. Chromosom-17-Veränderungen bei diploidem Hintergrund konnten in der SIOP-PNET4-Studie als ungünstiger prognostischer Faktor bei Standard-Risikopatienten identifiziert werden. Sehr aggressive Tumoren sind oft durch Amplifikationen von MYC-Genen und cytologischen Anaplasiezeichen gekennzeichnet (großzelliges und anaplastisches Medulloblastom).

ZNS-PNET umfassen Ependymoblastome (EBL, synomym: ETANTR, ETMR), Medulloepitheliome (MEP), ZNS-Neuroblastome, ZNS-Ganglioneuroblastome und ZNS-PNET, NOS („not otherwise specified“). Während EBL und MEP typische Amplifikationen des C19MC MikroRNA-Clusters und Zugewinne von Chromosom 2 zeigen, und zumeist mit ungünstigem klinischem Verlauf assoziiert sind, konnten bei den anderen Varianten Amplifikationen des MYCN-Gens als ungünstiger prognostischer Parameter identifiziert werden. Expressions- und Methylierungsanalysen können die Abgrenzung zu anderen Tumoren, insbesondere Gliomvarianten unterstützen. Pineoblastome ähneln biologisch Medulloblastomen, zeigen aber nicht die für letztere typischen Chromosom-17-Veränderungen oder Hedgehog/WNT-Aktivierungen. Auch Ependymome des Kindesalters lassen sich genetisch und biologisch subklassifizieren, wobei sich supratentorielle, infratentorielle und spinale Ependymome hinsichtlich ihrer Expressionsmuster, Genetik und Methylierungsmuster unterscheiden. In der überwiegenden Zahl der supratentoriellen Ependymome wurde eine Aktivierung des NFkBSignalweges durch RELA-Genfusionen gefunden; ein größerer Teil der infratentoriellen Ependymome zeigt charakteristische Methylierungsveränderungen. Hinsichtlich der Prognose ist die histopathologische Gradierung im Gegensatz zum Resektionsausmaß wenig aussagekräftig. Strukturelle chromosomale Veränderungen, insbesondere Zugewinne von Chromosom 1q konnten dagegen als unabhängiger prognostischer Faktor identifiziert werden.
Eine präzise molekulare und histologische Klassifikation wird letztlich die Patientenstratifizierung in zukünftigen Therapiestudien unterstützen und eine Selektion von Patienten für zielgerichtete Therapieansätze ermöglichen.

Gefördert durch die Deutsche Kinderkrebsstiftung und das Bundesministerium für Bildung und Forschung.

\section{Hochrisiko-Medulloblastom: Erfahrungen aus HIT2000}

\section{Mynarek', K. von Hoff', R.D. Kortmann'2, S. Rutkowski' et al.}

'Universitätsklinikum Hamburg-Eppendorf, Klinik für Pädiatrische Hämatologie und Onkologie, Hamburg, ${ }^{2}$ Universität Leipzig, Klinik für Radioonkologie, Leipzig

Die Kenntnis von Risikofaktoren hat die Einschätzung der individuellen Prognose von Medulloblastom-Patienten deutlich verbessert, allerdings sind bei Patienten mit hohem Risiko die Möglichkeiten einer Therapieintensivierung limitiert.

Bei Patienten <4 Jahre ist der histologische Subtyp der beherrschende Prognosefaktor. Desmoplastische Medulloblastome (DMB) und Medulloblastome mit extensiver Nodularität (MBEN) werden unabhängig vom Metastasierungsstatus als Niedrigrisikoerkrankung (LR), klassische (CMB), anaplastische (AMB) oder großzellige Medulloblastome (LCMB) als Hochrisikoerkrankung (HR) angesehen.

Bei Mo HR-Patienten konnte in HIT20oo nicht gezeigt werden, dass die Hinzugabe einer lokalen Radiotherapie zur postoperativer Chemotherapie zu einem verbesserten Überleben führt (3-Jahres-EFS $37 \pm 10 \%$ vs. $29 \pm 12 \%$; $p=0,823)$. Rezidive nach lokaler Radiotherapie waren häufiger metastatisch (5/17 vs. 13/13 metastatische Rezidive). Daher empfehlen wir derzeit bei HR-Mo die Chemotherapie als einzige postoperative Primärtherapie und die kraniospinale Radiotherapie (CSI) nur bei persistierender Erkrankung bzw. im Rezidiv. Intraventrikuläres MTX sollte während der Chemotherapie nicht gegeben werden, da ein großer Anteil der Patienten eine CSI benötigt und intraventrikuläres MTX den negativen Einfluss der CSI auf das neurokognitive Outcome verstärkt. Kinder mit $\mathrm{CMB} / \mathrm{AMB} / \mathrm{LCMB}$ erhalten bereits ab einem Alter von 3 Jahren eine primäre CSI gemäß PNET5-Konzept.

Bei M+ Patienten konnte in HIT2ooo gezeigt werden, dass eine intensivierte Induktionstherapie vor Hochdosischemotherapie \pm CSI das Ansprechen und das Überleben verbessert (3-Jahres-EFS $56 \pm 9 \%$ vs. $29 \pm 10 \% ; \mathrm{p}=0,019)$. Trotz relevanter Toxizität sehen wir deshalb die intensivierte Induktion als Standard an.

Bei Patienten >4 Jahren erfolgte die Therapiestratifizierung in HIT2ooo nur nach Metastasierungsgrad. Patienten mit metastatischem Medulloblastom erhielten einarmig eine Therapiesequenz aus SKK-Chemotherapie, hyperfraktionierter Radiotherapie und 4 Blöcken Erhaltungschemotherapie. Dieses Konzept führte zu einem signifikant besseren Überleben im Vergleich zur Vorläuferstudie HIT' 91 (5-Jahres EFS $47 \pm 6$ vs. $60 \pm 5 \% ; \mathrm{p}=0,246,5$-Jahres-OS $55 \pm 6$ vs. $73 \pm 4 \%$; $\mathrm{p}=0,037)$. Prognostische Faktoren für das Überleben waren histologischer Subtyp, Aktivierung des WNT-Signaltransduktionsweges und Ansprechen auf die Induktionschemotherapie. Eine internationale Folgestudie unter Einbeziehung dieser Faktoren ist in Vorbereitung.

Ausblick. Eine Weiterentwicklung von Stratifizierung und Therapie wird in internationalen Studien erfolgen. Die Einsendung von Tumormaterial ist hierbei essenziell.

Gefördert durch die Deutsche Kinderkrebsstiftung. 


\section{Therapie von CNS PNET und Pineoblastomen: Ergebnisse der HIT2000-Studie und einer internationalen Metaanalyse}

\section{K. von Hoff', M. Kool', T. Pietsch', S. Rutkowski' et al.}

${ }^{1}$ Klinik für pädiatrische Hämatologie und Onkologie, Universitätsklinikum Hamburg Eppendorf, Hamburg, '2Deutsches Krebsforschungszentrum, Heidelberg, ${ }^{3}$ Institut für Neuropathologie, Universitätsklinikum Bonn, Bonn

CNS-PNET und Pineoblastome sind seltene, heterogene Hirntumoren, die in HIT20oo und anderen Studien in gemeinsamen, multimodalen Therapiekonzepten behandelt wurden. Seit Beginn der Rekrutierung zu HIT20oo wurde die zugrundeliegende WHO-Klassifikation weiterentwickelt. Umfangreichere immunhistochemische Standardfärbungen ermöglichen inzwischen eine verbesserte Abgrenzung von CNS-PNETSubgruppen wie dem Ependymoblastom sowie von Tumoren anderer ontogenetischer Herkunft.

In HIT20oo wurden 121 Patienten mit CNS-PNET oder Pineoblastom behandelt. Bei den jungen Patienten bestätigte sich der positive Einfluss einer intensivierten Therapie mit Hochdosischemotherapie und autologer Stammzellgabe. Bei den älteren Patienten war die Einführung einer primären SKK Chemotherapie vor der hyperfraktionierten Radiotherapie für nicht metastasierte Patienten nicht mit einem deutlichen Vorteil assoziiert.

Im Rahmen einer Metaanalyse wurden klinische Originaldaten von 495 Patienten aus 11 Ländern gesammelt. Fünf-Jahres progressionsfreies (PFS) und Gesamtüberleben waren $36 \pm 2 \%$, und $41 \pm 2 \%$. Die Lokalisation des Rezidivs war primär lokal bei CNS-PNET ohne weitere Spezifizierung (CNS PNETnos) im Vergleich zu häufigeren distanten/kombinierten Rezidiven bei Pineoblastomen. Unabhängige Risikofaktoren für das PFS war in der Gruppe der Pineoblastome das Alter bei Diagnose und in der Gruppe der CNS-PNETnos das initiale Staging. Ein Viertel der Patienten waren Therapieversager, die eine frühe Progression entwickelten. Bei den übrigen Patienten waren die Applikation von Radiotherapie, die Therapie mit einer Hochdosischemotherapie, sowie ein gutes Therapieansprechen mit verbesserten Überlebensraten assoziiert. Eine histologische Reevaluation und molekulare Profilanalysen sind in diesem Kollektiv geplant.

Junge Patienten mit CNS PNET oder Pineoblastom sollten eine intensivierte Chemotherapie mit einer Hochdosischemotherapie erhalten. Bei älteren nicht metastasierten Patienten erscheint eine primäre Radiotherapie sinnvoll. Um auch in dieser Gruppe von seltenen Tumoren einen Fortschritt im Verständnis der molekularen Eigenschaften und möglichen Subgruppen zu erreichen, ist eine Sammlung von Tumormaterial im Zusammenhang mit einer guten klinischen Dokumentation (im Rahmen des HIT-MED Registers, vormals HIT-20oo interim) von hoher Wichtigkeit.

Gefördert durch die Deutsche Kinderkrebsstiftung.

\section{Risikoadaptierte Behandlung von intrakraniellen Ependymomen: Ergebnisse von HIT2000 und Ausblick auf SIOP-Ependymoma II}

\section{K. von Hoff', M. Mynarek', R.D. Kortmann², S. Rutkowski' et al. \\ 'Universitätsklinikum Hamburg-Eppendorf, Klinik für Pädiatrische Hämato- logie und Onkologie, Hamburg, ${ }^{2}$ Universität Leipzig, Klinik für Radioonkologie, Leipzig}

In Deutschland werden jährlich ca. 30 Patienten mit Ependymom der HIT-MED-Studienzentrale gemeldet. Die Standardtherapie für Patienten mit nichtmetastasiertem, komplett reseziertem Ependymom ist die lokale Radiotherapie. Der Stellenwert einer adjuvanten Chemotherapie sowie die optimale Therapie bei prognostisch ungünstigem, inoperablem Resttumor sind weiterhin unklar.
In HIT20oo wurden 296 nichtmetastasierte Studienpatienten mit referenzpathologisch bestätigtem Ependymom in 63 Zentren behandelt. Das PFS bei Patienten ohne Resttumor unterschied sich nicht zwischen jungen Patienten, die primär zur Verzögerung der Radiotherapie mit einer SKK Chemotherapie behandelt wurden (Alter $<4$ Jahre, $n=79$, 3 -Jahres-PFS $73 \pm 5 \%)$ und älteren Patienten, die primär eine lokale Radiotherapie erhalten haben (Alter $\geq 4$ Jahre, $n=124$, 3-Jahres-PFS $75 \pm 4 \%$; $\mathrm{p}=0$,94). Die Einführung einer primären Chemotherapie für Patienten mit postoperativem Resttumor verbesserte die Überlebenszahlen nicht. Wenn eine Zweitoperation eine komplette Resektion erreichte, war das Überleben besser als bei inoperablem oder nicht operiertem Resttumor. Metastasierte Erkrankungen waren selten und mit einer schlechten Prognose assoziiert ( $\mathrm{n}=13$, 3-Jahres-PFS $39 \pm 14 \%)$. Bei 95 in den Vorstudien behandelten Patienten zeigte sich im Langzeitverlauf ein deutlicher Abfall des Gesamtüberlebens (3-Jahres-OS $74 \pm 5 \%$ vs. 15-Jahres-OS $47 \pm 6 \%)$.

Neben Resttumor und Tumorlokalisation waren in HIT2ooo die genomische Gruppe und der 1q Zugewinn prognostische Faktoren. Eine pathologische Reevaluation von älteren Tumorproben, sowie weitere biologische Analysen sind geplant.

Die internationale, multizentrische SIOP-Ependymoma-II-Studie wird vermutlich ab 2015 in Deutschland zur Rekrutierung öffnen und sieht eine randomisierte Evaluation der Effektivität einer Erhaltungschemotherapie nach lokaler Bestrahlung für Kinder ab 1 Jahr vor. Weitere Fragestellungen von SIOP-Ependymoma II sind die Evaluation von molekularen Markern sowie die Therapieoptimierung für sehr junge Patienten (Chemotherapie \pm Valproinsäure) und für Patienten mit postoperativem Resttumor (neurochirugisches Referenzpanel, postoperative Chemotherapie \pm Methotrexat; hypofraktionierter Boost).

Zusammengefasst erscheint für die klinische Betreuung der Patienten v. a. die Evaluation einer Zweitoperation bei postoperativem Resttumor relevant. Um langfristig die therapeutischen Konsequenzen einer verbesserten Stratifizierung zu evaluieren und um Targets für innovative Therapien zu identifizieren, ist die Sammlung von Tumormaterial essenziell.

Gefördert durch die Deutsche Kinderkrebsstiftung.

\section{Sichelzellerkrankung}

\section{Die neue GPOH-Registerstudie „Sichelzellkrankheit"}

\section{H. Cario', R. Grosse', A. Jarisch', A.E. Kulozik', J. Kunz ${ }^{4}$, S. Lobitz}

Universitätskliniken für Kinder- und Jugendmedizin ${ }^{\mathrm{U} U \mathrm{Im}},{ }^{2} \mathrm{Hamburg},{ }^{3} \mathrm{Frank}-$ furt, ${ }^{4}$ Heidelberg und ${ }^{5}$ Berlin. Alle Autoren haben in gleichem Maße zu der Arbeit beigetragen.

Im September 2012 hat die GPOH-Mitgliederversammlung ein Konsortium aus fünf Universitätskliniken (Ulm, Frankfurt, Hamburg, Heidelberg und Ulm) mit der Leitung der neuen Registerstudie „Sichelzellkrankheit" beauftragt.

Kernstück der neuen Studie ist ein MARVIN-basiertes Patientenregister, in das alle in Deutschland und Österreich lebenden Patienten gemeldet werden. Zeitgleich mit dem Start des Registers wird eine von der Studienleitung in Zusammenarbeit mit einer pädiatrisch/internistischhämatologischen Expertengruppe verfasste, neue S2-AWMF-Leitlinie „Sichelzellkrankheit" verabschiedet, die detaillierte Empfehlungen für die Diagnostik und Therapie der Grunderkrankung sowie möglicher Komplikationen und eine Übersicht über die diesen Empfehlungen zugrundeliegende Literatur enthält. Dabei wird insbesondere auch den Entwicklungen in der Diagnostik und Prophylaxe zerebrovaskulärer Veränderungen sowie im Bereich der Therapie mit Hydroxycarbamid sowie bezüglich der Indikationen zur allogenen Stammzelltransplantation Rechnung getragen. 
Im Register werden Daten zur klinischen Situation sowie Laborergebnisse zu Beginn und im Krankheitsverlauf erfasst, die auf der Basis der Empfehlungen der Leitlinie erhoben werden. Das Register soll eine wichtige Grundlage für die Verbesserung der Patientenversorgung bilden und künftig auch eine Basis für wissenschaftliche Projekte bieten. Eine wesentliche Aufgabe der Studienleitung ist außerdem die Harmonisierung der Aktivitäten hinsichtlich der Stammzelltransplantation bei Sichelzellkrankheit in Deutschland. Eine Registerstudie „HSZT bei Sichelzellkrankheit" befindet sich gegenwärtig in der Planungsphase, in die nach einem ersten Treffen der Studiengruppe mit Vertretern interessierter Transplantationszentren im Januar 2014 zahlreiche Kliniken in Deutschland, Österreich und der Schweiz involviert sind.

Im Zentrum des Interesses der Studienleitung steht zudem die Klärung der Frage, ob die Einführung eines Neugeborenenscreenings auf Sichelzellkrankheiten auch in Deutschland sinnvoll und möglich ist. Dazu werden zunächst epidemiologische Daten benötigt. Ein erstes Pilotprojekt aus Berlin ist bereits publiziert, Daten aus Heidelberg und Hamburg folgen in Kürze. Die Berliner Daten zeigen, dass die Sichelzellkrankheit - zumindest im großstädtischen Bereich, wo ein großer Teil der Bevölkerung einen Migrationshintergrund hat - in einer Häufigkeit auftritt, welche die der häufigsten, bislang im Neugeborenenscreening erfassten Störungen übersteigt.

\section{Palliativmedizin}

\section{Akzeptanz und Effektivität der spezialisierten ambulanten Palliativversorgung von Kindern - eine prospektive Studie}

\section{G. Groh', U. Bender', G.D. Borasio' , M. Führer?}

'Koordinationsstelle Kinderpalliativmedizin, Dr. von Haunersches Kinderspital, Klinikum der Universität, München, ${ }^{2}$ Service de Soins Palliatifs, Centre Hospitalier Universitaire Vaudois (CHUV), Universität Lausanne, Schweiz

Hintergrund. Seit 2007 haben Kinder mit fortgeschrittenen lebensbegrenzenden Erkrankungen einen Anspruch auf spezialisierte ambulante pädiatrische Palliativversorgung (SAPPV). Ziel der prospektiven, nichtrandomisierten Studie war die Evaluation der Akzeptanz und Effektivität der SAPPV.

Methodik. Alle zwischen April 2011 und Juni 2012 durch das SAPPVTeam am Klinikum der Universität München behandelten Patienten waren für die Studie geeignet. Ausschlusskriterien waren Ablehnung durch Eltern oder Patient, psychosoziale Krisensituationen, oder unzureichende sprachliche oder intellektuelle Fähigkeiten. Mittels zweier Fragebögen wurde der primär pflegende Elternteil vor und während der Versorgung mittels standardisierter Instrumente und eigens entwickelter Fragen u. a. zur eigenen Lebensqualität (QoL) und der QoL des Patienten, sowie zur Symptombelastung, zur eigenen psychischen Belastung, zu Angst und Depression (HADS) sowie zur Belastung durch die Pflege (HPS) befragt.

Ergebnisse. Von 43 Familien, die im Untersuchungszeitraum neu in der SAPPV betreut wurden, konnten 40 in die Studie aufgenommen werden. Das mediane Intervall zwischen der ersten und zweiten Befragung war 8 Wochen. Das Alter der Kinder betrug im Median 6 Jahre (Range: 1 Monat bis 18 Jahre), $57 \%$ waren männlich, $25 \%$ hatten maligne Erkrankungen. In 37 Familien beantworteten die Mütter die Fragebögen, aufgrund ihres jungen Alters oder schwerer kognitiver Beeinträchtigungen konnten nur 3/40 Kindern selbst befragt werden. Die Betreuung durch das SAPPV-Team führte zu einer Verbesserung der Symptome des Kindes und seiner QoL ( $\mathrm{p}<\mathrm{0}, 001$; Fremdeinschätzung durch die Eltern). Auch die QoL des pflegenden Elternteils verbesserte sich signifikant (QOLLTI-F, $\mathrm{p}<0,001$ ), während sich die psychische Belastung reduzierte (HADS, p<0,001; HPS, $\mathrm{p}<0,001$ ).
Diskussion. Die Versorgung durch ein SAPPV-Team kann zu einer erheblichen Verbesserung in der Lebensqualität der Kinder und ihrer Eltern führen, und die psychische Belastung und die Belastung der Eltern durch die Pflege ihrer schwerstkranken Kinder deutlich reduzieren.

\section{Advance Care Planning (ACP) in der Pädiatrie: Erfahrungen und Bedürfnisse betroffener Eltern}

\section{J.D. Lotz', R.J. Jox², G.D. Borasio ${ }^{3}$, M. Führer}

'Koordinationsstelle Kinderpalliativmedizin, Dr. von Haunersches Kinderspital, Klinikum der Universität, München, ${ }^{2}$ Institut für Ethik, Geschichte und Theorie der Medizin, Ludwig-Maximilians Universität, München, ${ }^{3}$ Service de Soins Palliatifs, Centre Hospitalier Universitaire Vaudois (CHUV), Universität Lausanne, Schweiz

Hintergrund. ACP erleichtert Behandlungsentscheidungen, die sich am Willen des Patienten orientieren. In der Erwachsenenmedizin wurde ACP vielerorts erfolgreich umgesetzt und evaluiert. In der Pädiatrie gibt es wenige Initiativen und Studien zu ACP. Konzepte aus der Erwachsenenmedizin können nicht uneingeschränkt übertragen werden. Besonderheiten in der Pädiatrie bestehen in psychosozialer, ethischer und juristischer Hinsicht. Ist das Kind nicht einwilligungsfähig, treffen Eltern stellvertretende Entscheidungen zum Wohle des Kindes. Ergebnisse einer kürzlich publizierten systematischen Übersichtsarbeit zeigen, dass Eltern ACP als hilfreich erleben. Fehlende Akzeptanz und Unsicherheit im Rettungsdienst, in Schulen und im sozialen Umfeld erschweren jedoch die Umsetzung schriftlich festgelegter Behandlungsentscheidungen. Ziel der Studie ist die Erfassung der Erfahrungen und Bedürfnisse betroffener Eltern in Bezug auf ACP.

Methodik. Es wurden semistrukturierte Interviews mit Eltern verstorbener Kinder durchgeführt. Die Teilnehmer wurden nach der Methode des theoretischen Samplings ausgewählt, mit dem Ziel einer maximalen Variation hinsichtlich Diagnose, Alter bei Versterben, Sterbeort, Versorgung durch ein Team der spezialisierten ambulanten pädiatrischen Palliativversorgung sowie elterlichem Geschlecht. Die Interviews wurden inhaltsanalytisch ausgewertet.

Ergebnisse. Eltern erleben frühzeitige Diskussionen über Krankheitsverlauf und Behandlungsoptionen als hilfreich und entlastend. Positive Effekte sind: Gefühl der Beteiligung, Sicherheit und Kontrolle, Gefühl gute Eltern zu sein, verbesserte elterliche Entscheidungskompetenz in Akutsituationen. Wichtige Aspekte von ACP sind: frühzeitiger Beginn und regelmäßige Folgegespräche, umfassende Informationen, „shared decision-making" mit Beteiligung des Kindes, Vorbesprechung möglicher Krisen und konkrete Notfallplanung, 24-h-Verfügbarkeit eines primären Fallverantwortlichen, Widerrufbarkeit schriftlicher Festlegungen, gute Kooperation zwischen den örtlichen Versorgungspartnern. Die psychische Vorbereitung spielt für Eltern eine größere Rolle als die Dokumentation der ACP-Ergebnisse in einer schriftlichen Verfügung. Erlebte Schwierigkeiten in Bezug auf ACP gehen auf das Fehlen systematischer regionaler und strukturübergreifender Ansätze zurück. Diskussion. Aus Sicht der Eltern besteht ein Bedarf an systematischem ACP. Die Ergebnisse dieser Studie liefern wichtige Anhaltspunkte für die Entwicklung zukünftiger ACP-Programme. 


\section{Fortschritte in den palliativen Versorgungsstrukturen für onkologisch erkrankte Kinder - Ergebnisverbesserung für die Familien?}

\section{P. Schmidt, C. Hasan, B. Zernikow}

Deutsches Kinderschmerzzentrum und Kinderpalliativzentrum Datteln, Vestische Kinder- und Jugendklinik Datteln, Lehrstuhl für Kinderschmerztherapie und Pädiatrische Palliativmedizin, Universität Witten/Herdecke, Datteln

Hintergrund. Die Versorgung krebskranker Kinder in der Lebensendphase (LEP) erfolgt über individuelle Modelle der kinderonkologischen Abteilungen (koA). Weitere erkennbare Fortschritte in der pädiatrischen Palliativversorgung sind die Einführung der spezialisierten ambulanten Palliativversorgung (SAPV) in das SGB V sowie die wachsende Anzahl an Kinderhospizen. Trotz dieser positiven Entwicklungen ist die Versorgung krebskranker Kinder in der LEP suboptimal. Viele der Kinder leiden in der LEP unter Schmerzen und Erschöpfung und versterben nicht an dem gewünschten Ort

Ziel. Ziel der von der Deutschen Kinderkrebsstiftung geförderten Studie ist es darzustellen, ob betroffene Familien den Fortschritt in den pädiatrisch palliativen Versorgungsstrukturen wahrnehmen und wie sie davon profitieren.

Methode. In den Jahren 2003/04 und 2010/11 wurden je 48 Eltern von Kindern, die vor 5 Jahren an Krebs verstorben sind, mittels standardisiertem Fragebogen interviewt. Die Eltern wurden über teilnehmende koA in NRW rekrutiert (2003/o4 $n=6 ; 2010 / 11 n=16)$. In den 16 koA der zweiten Stichprobe wurden zudem Strukturdaten erhoben.

Ergebnisse. Nach Aussage der Eltern litten die Kinder in der LEP unter Schmerzen und Erschöpfung. Nur wenige Symptome wurden erfolgreich behandelt. Knapp drei Viertel der Kinder der 2. Stichprobe sind nach Aussage der Eltern zu Hause versorgt worden. Lediglich 50\% sind zu Hause verstorben, obwohl 93\% der Eltern, deren Kind im Krankenhaus verstorben ist, sich „zu Hause“ als Sterbeort gewünscht hätten. Die Strukturdaten zeigen, dass ein Großteil der koA ihren Patienten schon im Jahr 2005 eine häusliche palliative Versorgung angeboten hat. Trotz eines Anstiegs an Mitarbeitern mit Weiterqualifikation im Bereich „Palliativversorgung" gab es im Jahr 2010 in 30\% der koA keinen Mitarbeiter mit einer solchen Qualifikation.

Schlussfolgerungen. Trotz der erkennbaren positiven Entwicklungen der palliativen Versorgungsstrukturen für Kinder mit Krebs muss die häusliche Palliativversorgung für diese Kinder weiter optimiert und zu einem flächendeckenden Angebot ausgebaut werden.

1. Schmidt P, Otto M, Hechler T, Metzing S, Wolfe J, Zernikow B (2013) Did increased availability of paediatric palliative care lead to improved palliative care outcomes in children with cancer? Journal of Palliative Medicine 16(9):1034-1039

2. von Lützau P, Zernikow B (2013) Strukturen pädiatrischer Palliativversorgung in kinderonkologischen Abteilungen in Nordrhein-Westfalen. Zeitschrift für Palliativmedizin 14(3):114-120

2. von Lützau P, Otto M, Hechler T, Metzing S, Wolfe J, Zernikow B (2012) Children dying from cancer: Parents' perspectives on symptom, quality of life, characteristics of death and end-of-life decision. Journal of Palliative Care 28(4):274-281

\section{Freie Beiträge}

\section{Phase I/II Vorinostat für Kinder mit rezidivierten malignen Erkrankungen: Interimsanalyse}

\author{
O. Witt ${ }^{1,2}$, J. Burhenne ${ }^{3}$, R. Witt ${ }^{1,4}$, T. Milde ${ }^{1,2}$, C. van Tilburg ${ }^{2,4}$, A. Seitz ${ }^{5,}$ \\ J.-P. Schenk ${ }^{6}$, A. Eisenmenger ${ }^{4}$ I. Karapanagiotou-Schenkel ${ }^{4}$
}

${ }^{1}$ KKE Pädiatrische Onkologie $\left(\mathrm{G}^{340}\right)$, Deutsches Krebsforschungszentrum (DKFZ), Heidelberg, ${ }^{2}$ Sektion Pädiatrische Hirntumore, Klinik Pädiatrie ${ }^{3}$, Universitätsklinikum Heidelberg, Heidelberg, ${ }^{3}$ Abteilung Klinische Pharmakologie und Pharmakoepidemiologie, Universitätsklinikum Heidelberg, Heidelberg, ${ }^{4} \mathrm{NCT}$ Zentrum für klinische Studien, Nationales Centrum für Tumorerkrankungen (NCT), Heidelberg, ${ }^{5}$ Abteilung Neuroradiologie, Universitätsklinikum Heidelberg, Heidelberg, ${ }^{6}$ Sektion Kinderradiologie, Abteilung Radiologie, Universitätsklinikum Heidelberg, Heidelberg

Hintergrund. Histondeacetylasen (HDACs) sind Schlüsselenzyme, die Apoptose, Differenzierung, Zellzyklus und Chemotherapieresistenz über epigenetische und zytoplasmatische Signalwege kontrollieren. In präklinischen Vorarbeiten konnten wir zeigen, dass einzelne Mitglieder der HDAC-Familie 1-11 spezifische onkogene Funktionen in pädiatrische Tumormodellen ausüben und in Hochrisikotumoren hoch exprimiert sind. In der multizentrischen Phase-I/II-Studie werden Kinder und Jugendliche mit rezidivierten soliden Tumoren, Lymphomen und Leukämien mit dem HDAC-Inhibitor Vorinostat behandelt.

Methoden. Kinder und Jugendliche im Alter von 3 bis 18 Jahren mit rezidivierten malignen Erkrankungen werden im Rahmen einer multizentrischen, nichtkontrollierten Studie nach einem intraindividuellen Dosiseskalationskonzept behandelt, um für jeden Patienten die optimale Dosis hinsichtlich Verträglichkeit und Wirksamkeit zu erreichen. Primäre und sekundäre Endpunkte sind Bestimmung der Dosis, dosislimitierende Toxizitäten, PK, PD, Biomarkerbestimmung und Responserate.

Ergebnisse. Der erste Patient wurde 05/2012 rekrutiert. Aktuell wurden 32 Patienten in 10 pädiatrisch-onkologischen Zentren der GPOH in die Studie aufgenommen. Die häufigsten Entitäten sind hochgradige Hirntumoren. Individuell maximal tolerable Dosierungen (MTD) reichen von 130 bis $640 \mathrm{mg} / \mathrm{m} 2 /$ Tag. Die häufigste dosislimitierende Toxizität ist die Thrombozytopenie. Grad 3 und 4 SAEs betreffen Einzelfälle von Kopfschmerzen, Müdigkeit, Erbrechen, Infektionen und Thrombozytopenie. Bisher wurde kein SUSAR gemeldet. Bisher konnten bei 6 in MTD behandelten Patienten eine zentrale Responsebeurteilung durchgeführt werden, die in 3/6 Fällen eine partielle Response nach RECIST dokumentiert (Diagnosen: DIPG, GBM, SETTLE). Die längste Nachbeobachtung beträgt derzeit 20 Monate eines Patienten, der weiterhin die Vorinostat-Therapie erhält. PK, PD und Biomarker-Analysen werden derzeit durchgeführt.

Schlussfolgerung. Die Interimsanalyse zeigt, dass ein intraindividuelles Dosiseskalationskonzept in dieser Patientenpopulation machbar ist, welches dem einzelnen Patienten erlaubt, seine optimale Dosis hinsichtlich Toxizität und Wirksamkeit zu erhalten. Die bisherigen Toxizitätsdaten zeigen ein insgesamt moderates und reversibles Toxizitätsprofil mit Thrombozyopenie als unerwünschte Wirkung. Einzelne Patienten, die eine hohe Dosiseskalationsstufe erreicht haben, zeigen eine Response. Die Rekrutierung wird voraussichtlich in 1 Jahr abgeschlossen sein.

Diese Investigator-initiierte Studie wird finanziell von der Deutschen Kinderkrebsstiftung unterstützt und die Studienmedikation von MSD zur Verfügung gestellt. 


\section{T-cells are not required for the pathogenesis of hemophagocytic lymphohistiocytosis in humans}

\section{S.F.N. Bode ${ }^{1,2}$, S. Ammann', K. Lehmberg ${ }^{3}$, S. Ehl' ${ }^{1,2}$ for the GPOH, the Histio- cyte Society and the IEWP of the EBMT \\ ${ }^{1}$ Centre of Chronic Immunodeficiency, University Medical Centre Freiburg, Germany, ${ }^{2}$ Centre for Pediatrics and Adolescent Medicine, University Medical Centre Freiburg, Germany, ${ }^{3}$ Department of Haematology and Oncology, Chil- dren's Hospital, University of Hamburg, Germany}

Background. Hemophagocytic lymphohistiocytosis (HLH) is a lifethreatening syndrome of severe hyperinflammation. Impaired release of perforin-containing granules by T-/NK-cells is a key factor in hereditary $\left(1^{\circ}\right) \mathrm{HLH}$. Mouse models of $1^{\circ} \mathrm{HLH}$ indicate that T-cells and IFN- $\gamma$ are essential for disease pathogenesis. Whether T-/NK-cells are required for other forms of HLH in humans is unclear

Methods. Primary immunodeficiencies (PID) provide an excellent opportunity to study the role of immune cells and mediators in immune-mediated diseases. We collected the experience in the treatment of patients with PID except cytotoxicity disorders or XLP, who developed HLH, from the GPOH, the Histiocyte Society and the IEWP of the EBMT and added cases from the literature.

Results. 46 patients with PID had at least one episode of HLH. 14 patients had severe combined immunodeficiency ( 9 with $<100$ T-cells), 11 had partial T-cell deficiencies. Most of these HLH episodes were triggered by viruses. 11 patients had HLH in the context of chronic granulomatous disease, mainly associated with bacterial infection. 10 patients had other ID. Patients with T-cell deficiency showed significantly lower soluble IL-2 receptor ( $\mathrm{sCD}_{25}$ ) levels and higher ferritin levels than patients with cytotoxicity defects. Lower $\mathrm{sCD}_{25}$ levels were also observed in patients with infection-induced $2^{\circ} \mathrm{HLH}$ compared to patients with $1^{\circ} \mathrm{HLH}$.

Discussion. Our results are consistent with the concept of a T-cell dependent and a T-cell independent pathway of HLH pathogenesis. T-cells appear crucial for $1^{\circ} \mathrm{HLH}$, but are not required for the development of HLH in general and appear less active in many patients with $2^{\circ} \mathrm{HLH}$. Conclusion. HLH is a heterogeneous syndrome representing a common endpoint of different pathways of pathogenesis. T-cell directed therapy might not appropriate for all patients with HLH.

\section{Haploidentische Transplantation bei X-linked SCID: eine Single-centre-Analyse von 34 Patienten}

\section{Hönig, D. Kleila, K.-M. Debatin, W. Friedrich, A. Schulz \\ Klinik für Kinder- und Jugendmedizin, Universitätsklinikum UIm}

Einleitung. X-linked SCID ist die häufigste genetische Entität aus der Gruppe der Schweren Kombinierten Immundefekte und wird durch Mutationen in der common-gamma-chain (IL2RG) der Interleukinrezeptoren für IL-2, $-4,-7,-9,-15$ und -21 verursacht. Bei fehlendem HLA-identischem Spender ist die haploidentische Transplantation eine kurative Therapieoption.

Studiendesign. Retrospektive Analyse aller Patienten mit X-SCID die zwischen 1982 und 2005 in der Klinik für Kinder- und Jugendmedizin Ulm ein HLA-nicht-identisches elterliches Transplantat erhielten.

Ergebnisse. Das „overall survival“ (OS) der 34 Patienten nach haploidentischer Transplantation lag bei 82\% (28/34) nach einem medianen Follow-up von 10,8 Jahren (1,4-24). Es kamen zwei Methoden der TZell-Depletion zum Einsatz: Schafserythrozyten/Sojabohnenlektin-Sedimentation von 1982 bis 1995 bei 20 Transplantaten, CD 34 Positivselektion/In-vitro-T-Zell-Depletion von 1996 bis 2005 bei 14 Transplantaten. Das OS war hiervon unabhängig bei $80 \%$ (16/20) für die erste und $86 \%$ (12/14) für die zweite Gruppe. 2o Patienten wurden vor Gabe des Transplantats konditioniert (OS 85\%), bei 14 wurde keinerlei Chemotherapie vor der Transplantation verabreicht (OS 79\%). Zwei Patienten verstarben aufgrund nachgewiesener Infektionen (BCG, EBV), bei vier Patien- ten, die aufgrund einer respiratorischen Insuffizienz verstarben, konnte kein Erreger identifiziert werden. Kein Patient verstarb aufgrund einer GvHD. Spender-T-Zellen konnten mit nur einer Ausnahme bei allen überlebenden Patienten nach einer einmaligen Stammzellgabe nachgewiesen werden. Bei zwei Patienten wurden 6 bzw. 12 Jahre nach der ersten Stammzellgabe eine erfolgreiche Retransplantationen mit Konditionierung durchgeführt. Die Indikationen hierzu ergaben sich aus rezidivierenden pulmonalen Infektionen trotz Immunglobulinsubstitution bzw. einer Autoimmunhepatitis. Stabile B-Zell-Funktionen ohne die Notwendigkeit einer dauerhaften Immunglobulinsubstitution fanden sich bei 14/17 (82\%) Langzeitüberlebenden nach Konditionierung, jedoch bei lediglich $3 / 11$ (27\%) ohne Konditionierung.

Schlussfolgerung. Für Patienten mit X-SCID ohne die Möglichkeit einer raschen Identifikation eines HLA-kompatiblen Spenders ist die haploidentische Transplantation mit und ohne Konditionierung eine therapeutische Option mit sehr guten Überlebenschancen. Die Wahrscheinlichkeit stabiler B-Zell-Funktionen nach Transplantation kann durch eine Konditionierung positiv beeinflusst werden.

\section{Feasibility, risk profile and diagnostic yield of stereotactic biopsy in the pediatric population}

\section{M.I. Ruge', T. Blau' ${ }^{2}$, C. Hamisch, ${ }^{1,3}$, T. Simon ${ }^{4}$}

'Department of Stereotaxy and Functional Neurosurgery, Centre of Neurosurgery, University of Cologne, , ${ }^{2}$ Department of Neuropathology, University of Cologne, ${ }^{3}$ Department of General Neurosurgery, Centre of Neurosurgery, ${ }^{4}$ Department of Pediatric Hematology and Oncology, University of Cologne, Germany

Objective. While stereotactic biopsies (SB) are widely accepted as routine diagnostic tool for unclear cerebral pathologies in adult patients, there is still a certain reluctance to apply this technique for the pediatric population. Probable reasons might be the localization of pathologies predominantly in highly eloquent regions (posterior fossa/midline structures) and/or a perceived elevation of other peri-operative risks. Here we present feasibility, risk profile and diagnostic yield of SB in one of the largest reported pediatric populations.

Methods. All pediatric patients (age <21) who underwent SB in our institution (1994-2013) were analyzed in detail retrospectively regarding success of performing the SB, diagnostic yield, and procedural complications. These parameters were correlated with localization (lobar vs. midline) and amount of biopsy material (1 vs. $\geq 2$ probes)

Results. 133 patients (median age 11.0 \pm 5.1 , range 1-20) underwent 148 SB's located lobar (41) and midline (97). Tissue was obtained in all cases (90.6\% $\geq 2$ probes) and was diagnostic in $93.2 \%$. In $3.4 \%$ a second SB procedure was successful. Mortality was zero and procedural morbidity was transient and low (5.4\%). These parameters were not influenced by localization or amount of biopsy material.

Conclusion. SB can be performed safely and with a high diagnostic yield in the pediatric population in specialized institutions. Under the aspect of providing tissue for advanced histopathological- immunological and molecular information to tailor more individualized treatment this well established technique deserves to be considered more often in the pediatric population.

\section{Skoliose bei Neuroblastom und Ganglioneurom: Tumor- oder Therapiefolge?}

\section{E. Piepenbrock', B. Krug ${ }^{2}$, K. Zarghooni ${ }^{3}$, F. Berthold ${ }^{7}$}

'Kinderonkologie, Klinik und Poliklinik für Kinder und Jugendmedizin, ${ }^{2}$ Institut und Poliklinik für Radiologische Diagnostik, ${ }^{3}$ Klinik und Poliklinik für Orthopädie und Unfallchirurgie, Uniklinik Köln, Köln

Fragestellung. Skoliose ist als Spätfolge nach Neuroblastom (NB) und Ganglioneurom (GN) bekannt, jedoch ist nicht klar, inwiefern der 
Tumor selbst neben Wirbelsäulenoperationen oder Radiotherapie zur Skolioseentstehung beiträgt. Diese Studie untersucht Prävalenz und Risikofaktoren der Skolioseentwicklung bei Patienten mit NB oder GN. Studiendesign. Ausgewertet wurden Daten von Patienten mit NB oder GN, die zwischen dem 01.01.1990 und dem 31.12.2006 in den deutschen Neuroblastomstudien registriert wurden. Daten von Patienten mit Skoliose wurden verglichen mit den Daten eines Kontrollkollektivs ohne Skoliose mit einer Nachbeobachtung von mindestens sechs Jahren $(n=1698)$. Ergänzend wurden Kernspintomographien und Röntgenbilder von Patienten mit Skoliose zentral begutachtet.

Ergebnisse. Insgesamt 108 von 2672 Patienten zeigten eine Skoliose (4\%; 96/1588 NB; $12 / 110$ GN). Das mediane Alter bei Erstdiagnose der Skoliose betrug 6,5 Jahre (Range: 0-17 Jahre), der mediane Cobb-Winkel 24,2 ${ }^{\circ}$ $\left(10,2^{\circ}-96,8^{\circ} ; n=39\right)$. Der Tumor fand sich bei 71/96 Patienten an der Konvexität der Skoliose $(\mathrm{p}=0,004)$. Im Vergleich zur Kontrollkohorte wiesen Patienten mit Skoliose häufiger ein Stadium 3 nach INSS, (40\% vs. 17\%; $\mathrm{p}<0,001$ ), thorakale Tumore ( $55 \%$ vs. $14 \%$; $\mathrm{p}<0,001$ ) oder eine intraspinale Beteiligung ( $57 \%$ vs. $9 \%$; $<<0,001$ ) auf. Dreißig Patienten zeigten eine Skoliose vor Operation oder Radiotherapie. Bei 4 Patienten führte die Skoliose zur Tumordiagnose, bei 11 Patienten war die Skoliose bei der Tumordiagnose auffällig und 15 Patienten entwickelten eine Skoliose während Chemotherapie oder Beobachtung. Achtundsiebzig Patienten entwickelten eine Skoliose nach Thorakotomie/Laparotomie $(n=29)$, nach Wirbelsäulen-Eingriffen $(n=28)$, nach Strahlentherapie $(n=13)$ oder nach Strahlentherapie und Wirbelsäulen-Eingriffen $(n=8)$. Bei Patienten mit Skoliosediagnose vor Operation oder Radiotherapie war der Tumor histologisch differenzierter als bei Patienten mit Skoliose nach Operation oder Strahlentherapie (GN/NB 9/21 vs. 3/75; p<0,001).

Schlussfolgerung. Risikofaktoren für die Skolioseentstehung sind thorakale Tumoren, Stadium 3 und intraspinale Beteiligung. Der Tumor ist hauptsächlich auf der konvexen Seite der Skoliose lokalisiert. Bei einem Teil der Patienten besteht eine Skoliose bereits zur Tumordiagnose oder entwickelt sich in der initialen Therapiephase. Tumoren dieser Gruppe zeigten häufiger eine reife Histologie (GN).

\section{The addition of gemcitabine to 131I-MIBG therapy in pediatric patients with relapsed or progressive neuroblastoma: initial results of an ongoing phase II trial}

\section{N.K.A. van Eijkelenburg', L.A. Tytgat', T. Simon', C.M. Zwaan', H.N. Caron'}

'Department of Pediatric Oncology, Academic Medical Centre Amsterdam, the Netherlands, ${ }^{2}$ Department of Pediatric Oncology and Hematology, Children's Hospital, University of Cologne, Cologne, Germany, ${ }^{3}$ Department of Pediatric Oncology, Erasmus Medical Centre, Rotterdam, the Netherlands

Aims. Despite intensive multimodal approaches prognosis of stage 4 neuroblastoma remains poor. 131I-metaiodobenzylguanidine (MIBG) is a targeted radiopharmaceutical with activity in patients with relapsed or refractory neuroblastoma. Gemcitabine is a known radiosensitizer with preclinical activity in neuroblastoma. Single-agent gemcitabine has proven tolerability in pediatric patients with solid tumors. This phase II trial aims to evaluate the efficacy and safety of two cycles of the addition of gemcitabine as radiosensitizer to 131I-MIBG therapy.

Methods. Patients $<18$ years with relapsed or progressive neuroblastoma and with MIBG-avid tumors were eligible. Adequate bone marrow function (platelet count of $>100 \times 109 / \mathrm{L} ;>75$ if $\mathrm{BM}$ involved) and adequate renal and hepatic function was required. Gemcitabine $375 \mathrm{mg} / \mathrm{m} 2$ was given on days 1 and 8 of a 28-day cycle. 131-MIBG was given on day 1 with a starting dose of $444 \mathrm{Mbq} / \mathrm{kg}$ and aiming at a whole body dose of 4 Gy after 2 cycles. Response will be assessed according the new International Neuroblastoma Response Criteria after each cycle. Gemcitabine dose will be escalated if at interim analysis toxicity is acceptable and efficacy is intermediate.
Results. To date, 6 patients (median age 11.o years; range 5.2-12.0 years) received $1(n=3), 2(n=2)$, or $3(n=1)$ cycles of therapy. Myelosuppression was the most common toxicity. 1 patient at gemcitabine $375 \mathrm{mg} / \mathrm{m} 2$ dose level had dose-limiting toxicity (DLT) with prolonged grade 4 thrombocytopenia. Objective responses (1 partial response) occurred in 1 of 3 $(33 \%)$ evaluable patients. In the other patients, 1 stable disease was observed.

Conclusion. In the treatment of relapsed patients, the addition of gemcitabine to 13II-MIBG therapy is tolerable at this dose-level. The study is ongoing to assess safety in additional patients and to preliminary assess clinical activity.

\section{Einsatz von a-Tocopherolacetat-Creme zur Therapie und Prävention von Hauttoxizitäten bei Kindern mit akuten Leukämien - eine Zusammenfassung von Einzelfällen}

\section{K. Waack, D. Reinhardt}

Medizinische Hochschule Hannover, Zentrum Kinderheilkunde und Jugendmedizin, Klinik für Pädiatrische Hämatologie und Onkologie, Kooperative AML-BFM Therapiestudien, Hannover

Hintergrund. Das Auftreten von Hauttoxizitäten ist bei Kindern, die eine Therapie mit Purinanaloga (Cytarabin, Fludarabin, Clofarabin) oder Kinaseinhibitoren (z. B. Sorafenib) erhalten, eine häufige und belastende Nebenwirkung. Deshalb sind nebenwirkungsarme vorbeugende oder therapeutische Optionen wünschenswert. $\alpha$-Tocopherolacetat (Mapisal ${ }^{\circ}$ Creme), ein apothekenpflichtiges Medizinprodukt, soll präventiv wirken.

Methoden. Zwischen 02/2013 und 02/2014 wurden acht Patienten (AML $\mathrm{n}=7$; ALL $\mathrm{n}=1$ ) begleitend zur Chemotherapie mit Fludarabin, Clofarabin oder Sorafenib mit $a$-Tocopherolacetat versorgt. Bei fünf Kindern wurde $a$-Tocopherolacetat therapeutisch eingesetzt, bei vier Kindern als Prophylaxe, davon eins nach vorausgegangener therapeutischer Anwendung. Die fünf Patienten mit therapeutischer Anwendung wiesen Exantheme am gesamten Körper auf $(n=2)$ oder entwickelten ein Hand-Fuß-Syndrom $(n=2)$. Ein Kind zeigte eine ausgeprägte generalisierte Erythrodermie, Juckreiz und starken Schmerzen (Hauttoxizität CTCAE Grad III) unter Clofarabintherapie. Im letzten Fall erfolgte die Therapie mit kortisonhaltiger Creme, Clemastin, einem Hydromorphon-Perfusor und $\alpha$-Tocopherolacetat Creme. Insgesamt vier Kinder, teils nach ausgeprägten Hauttoxizitäten in vorangegangenen Therapieelementen, $\boldsymbol{\alpha}$-Tocopherolacetat-Creme vorbeugend bekommen.

Ergebnisse. Bei drei Patienten besserte sich die Symptomatik bereits nach wenigen Tagen. Sowohl die Exantheme und der Juckreiz als auch das Hand-Fuß-Syndrom waren rückläufig. Bei einem Kind zeigte sich kein Effekt, da sich die Hauttoxizität als GvHD (Grad I) in Folge der vorangegangenen Stammzelltransplantation herausstellte. Der Patient, der die Creme zunächst therapeutisch und dann präventiv bekam, war subjektiv mit dem Ergebnis sehr zufrieden. Die Haut schälte sich zwar und sah dünner aus, offene Läsionen verheilten jedoch und traten nicht erneut auf. Alle drei Patienten, die die Creme vorbeugend erhalten hatten, zeigten unter der Therapie mit Clofarabin/Fludarabin in Kombination mit Cytarabin $(n=2)$ oder mit Sorafenib und Gemtuzumab ozogamicin keine Hautauffälligkeiten. Bei keinem Patienten wurden unerwünschte Nebenwirkungen durch die Creme beobachtet.

Schlussfolgerung. Insgesamt wurde der Einsatz von $\alpha$-Tocopherolacetat positiv eingeschätzt. Eine kontrollierte Anwendung soll in der AMLBFM-Studie prospektiv erfasst werden. 


\title{
Abstracts
}

\section{Epigenetische Veränderungen definieren Gruppe-A-Ependymome im Kindesalter}

\author{
H. Witt ${ }^{1,2}$, S.C. Mack ${ }^{3}$, S.M. Pfister, ${ }^{1,2}$, M.D. Taylor ${ }^{3}$
}

'Division of Pediatric Neurooncology, German Cancer Research Center (DKFZ), Heidelberg, Germany, ${ }^{2}$ Department of Pediatric Oncology, Hematology and Immunology, University Hospital Heidelberg, Germany, ${ }^{3}$ Division of Neurosurgery, Brain Tumour Research Centre, The Hospital for Sick Children, Toronto, ON, Canada

Hintergrund. Das Ependymom ist der dritthäufigste kindliche Hirntumor. Es konnte bereits gezeigt werden, dass Ependymome der hinteren Schädelgrube („posterior fossa“, PF) in zwei klinisch und molekular unterschiedliche Subgruppen eingeteilt werden können (Gruppe A und Gruppe B). Während Gruppe-B-Tumoren mit einer guten Prognose und einer Vielzahl von genomischen Aberrationen assoziiert sind, überleben nur etwas 20\% der Patienten mit einem Gruppe-A-Ependymom länger als 5 Jahre; diese Tumoren zeigen kaum genomische Veränderungen.

Methoden. Um genetische und epigenetische Hinweise für die Tumorentstehung dieser Tumoren zu entdecken, führten wir Whole-genome-, und Whole-exome-sequencing-Analysen $(n=47)$ sowie DNA-Methylierungsanalysen $(n=79)$ von Ependymomen der hinteren Schädelgrube durch. Basierend auf Consensus-clustering-Analysen der DNA-Methylierungsprofile konnten zwei unterschiedliche Subgruppen identifiziert werden, Gruppe A und B. Um weitere epigenetische Hinweise zu detektieren, wurden sechs Tumoren mittels whole genome bisulphite sequencing und elf Tumoren mittels $\mathrm{H}_{3} \mathrm{~K}_{2} 7 \mathrm{me}_{3}$ ChIP-seq analysiert.

Ergebnisse. Im Gegensatz zu anderen kindlichen Krebserkrankungen wiesen PF-Ependymome mit durchschnittlich 5 somatischen Mutationen eine sehr niedrige Mutationsrate auf. Zudem zeigten sich keinerlei wiederkehrende Mutationen. Die DNA-Methylierung war hingegen deutlich unterschiedlich ausgeprägt, sodass Gruppe-A-Ependymome einen $\mathrm{CpG}$ island methylator phenotype aufwiesen. Eine epigenetische Inaktivierung zeigte sich insbesondere bei Zielgenen des polycomb repressor complex 2 (PRC2), dieser reguliert Gene der Zelldifferenzierung via Tri-Methylierung von $\mathrm{H}_{3} \mathrm{~K}_{2} 7$ in vitro und in vivo. Es konnte gezeigt werden, dass Gruppe-A-Ependymome auf eine zielgerichtete Behandlung der DNA- oder $\mathrm{H}_{3} \mathrm{~K}_{27}$-Methylierung im präklinischen Modell in vitro und in vivo ansprechen.

Schlussfolgerung. Zusammenfassend konnte dargestellt werden, dass sich, basierend auf einer molekularen Rationale, epigenetisch modifizierende Medikamente als eine zukünftige Behandlungsoption für Patienten mit einem Gruppe-A-Ependymom erweisen könnten. Ein tatsächlicher Nutzen dieses Therapieansatzes muss in zukünftigen präklinischen und klinischen Studien weiter belegt werden. 